\title{
On the Energy Evolution in Gaseous Detonation Waves
}

\author{
A.A. Borisov, O.I. Mel'nichuk, A.R. Kasimov, B.A. Khasainov, K.Ya. Troshin and V. Kosenkov \\ N. Semenov Institute of Chemical Physics, Russian Academy of Sciences, Moscow 117977, Russia
}

\begin{abstract}
There is a definite inconsistency between the classical ZND theory of detonation and contemporary experimental observations and attempts to model real detonation waves. Nevertheless, the classical onedimensional model of detonation is still extensively used in interpreting measurements because of its simplicity and physical clarity. This naturally raises the questions, what does actually the classical model represent and how one can relate the real multidimensional wave structure to an effective onedimensional detonation? The answer to these questions is extremely important from the practical point of view, because it defines how simple the solution of the criticality problems in detonation could be (i.e. whether one has to solve the very cumbersome three-dimensional nonsteady gasdynamic problem with detailed kinetics of the chemical reaction comprising a few hundreds of elementary steps to predict such parameters as critical initiation energy, critical distances, and concentrations, or the solution of a simplified onedimensional gasdynamic problem with global chemical kinetic equations would suffice?).

The present communication discusses the following $t, s$ with the aim of answering partly the above questions. (1) An analysis of the detailed kinetic calculations of the heat evolution rate in some fuel-air mixtures and comparison of these results with experiments reveals that (a) in the majority of detonable mixtures heat release can be described by global equations with an accuracy quite sufficient for assessing the critical detonation parameters, (b) chemical kinetics shows no so-called "recombination" zones with an appreciable heat evolution that follow the main reaction zone, (c) the ratio between the induction and the explosion (within which the major fraction of the stored energy is evolved) times exceeds unity under the conditions corresponding to detonation waves in fuel-air mixtures, which contradicts the shock tube measurements. All these results can be reasonably explained by the fact that the chemical reaction in detonation waves (in shock waves as well) proceeds in a gas with a highly inhomogeneous distribution of the parameters (temperatures, pressures, and particle velocity) which gives rise to the so-called hot-spot mechanism of chemical reactions in gaseous and two-phase media. It is shown that the hot spot ignition is the main reason of instability of detonation waves. Nonsynchronous mixture ignition at different points produces the effect of extended heat evolution zone and reduces the effective induction zone.

Results of measurements of averaged and local parameters behind detonation waves in gases support the idea that the effective heat evolution rate in the onedimensional representation of the wave is much more complicated than that inherent in the chemical reaction alone, because it contains also a significant contribution of the kinetic and thermodynamic energy redistribution within the major reaction zone and downstream of it, which makes the onedimensional structure of detonation waves deviate significantly from the classical pattern leading to nonmonotonic heat release behind the shock front (the possibility of appearance of two or more effective sonic planes and heat rlease maxima).

However, a onedimensional analysis of marginal detonation waves has demonstrated that critical parameters can be estimated quite accurately within simplified models, which is attributable to the fact that the criticality is associated with local termination of the reaction within narrow stream tubes where the flow pattern resembles closely the ZND non-CJ detonation wave structure. Unlike the chemical reaction zone in detonations far away from the limit, which is shorter than the detonation cell size, the reaction zone in marginal detonations is comparable with the cell size.

The results of onedimensional calculations of critical diameters and minimal energies of direct initiation of detonation are compared with experiment.
\end{abstract}




\section{INTRODUCTION}

It is unambiguously proved both theoretically and experimentally that onedimensional detonation waves in gaseous and two phase (sprays or dusty gases) are virtually nonexistent. Nonetheless, the so-called Zeldovich-von Neumann-Döring theory developed originally for essentially onedimensional detonation waves is still extensively used in interpreting experimental data. This can be attributed to three reasons: first, the ZND model is physically clear and simple and always warrants adequate, at least qualitative, explanation of all experimental observations, second, the properties of non-marginal detonation waves are described quite adequately by this model, except the real structure of the von Neumann spike (which is actually of little importance in many cases), and, finally, calculations using threedimensional models are so scarce and cumbersome that can not be considered as a series basis for interpreting any experimental results. Moreover, the ZND model applies undoubtedly to individual stream tubes even in three-dimensional nonsteady detonation waves in the sense that in narrow stream tubes one can distinguish the initiating shock wave, von Neumann spike, and the surface where the reaction is terminated, but these "elementary" detonation waves are not necessarily $\mathrm{CJ}$ waves.

Thus, the state of art in the detonation theory is such that the overidealized ZND model is used by almost everybody, while the realistic three-dimensional model, although clearly formulated, is too inconvenient for common use, and there is actually no intermediate model or even general understanding how the averaged (over space and time) detonation parameters correlate with the three-dimensional wave structure. Of importance is also to find out how adequately the one-dimensional ZND model describes marginal detonation waves and critical conditions for detonation initiation. A priori, one can expect that this simplified approach is applicable to treating critical detonation phenomena and calculating the von Neumann spike structure but with some effective averaged kinetic of energy release behind the lead shock front (which may drastically differ from the pure chemical kinetics). This was tacitly assumed in many theoretical works (see, e.g., [1]), where a two-stage reaction or "recombination stage" were introduced in the computational model.

This paper is an attempt to cover the gap between the aforementioned models and to answer the questions how the ZND model could be applied (if so) to at least approximate calculations of the realistic detonation parameters and what kind of kinetic equations should be used in these calculations.

\section{HIGH TEMPERATURE REACTION KINETICS}

General analysis of reaction kinetics implies that the reactive mixture has homogeneous properties. Both experiment and calculations with detailed chemical kinetics show clearly an induction period during which the temperature in the system changes only little and a stage of progressively accelerated temperature rise.

First we consider the induction stage, because quite often it constitutes the major part of the overall reaction time and in many cases it is the only parameter which is reliably measured in shock tube experiments. Most of the reactions in detonation waves comprise a great many elementary steps even within the induction period, therefore Arrhenius plots of induction periods $t_{\text {ind }}$ are not necessarily straight lines in a wide temperature range. There are examples of both flattening (mostly for hydrocarbons) and steepening (e.g. for hydrogen mixtures) of the $t_{\text {ind }}$ versus $1 / T$ dependence at lower temperatures. Such a behavior of the Arrhenius plots is associated with a changeover of the dominant mechanisms or rate 
controlling steps within the same reaction scheme, and hence can be observed only in systems where concurrent processes with different effective activation energies take place. Fortunately, in practice the characteristic times of changes in the flow parameters (i.e. times available for the reaction) range in rather narrow limits, so that the Arrhenius plots of ignition delays in each particular case may be represented by straight lines with a fairly good accuracy. Therefore one can expect that this reaction stage can be described by a global equation of the Arrhenius type, at least for conditions relevant to detonation processes.

Induction period is only a part of the total time during which heat is released by a chemical reaction. Unfortunately, there is no simple analytical means for calculating $t_{\mathrm{e}}$, i.e. the time within which the major fraction of the energy stored in the system is evolved. Usually interpretation of detonation phenomena associated with chemical kinetics is based on consideration solely of induction periods. The characteristic feature of $t_{\text {ind }}$ is its high temperature sensitivity, therefore the analysis of the shock wave - reaction zone complex leads inevitably to a conclusion that this complex is unstable. In a number of theoretical approaches the heat release pattern is represented even by a step-wise function. Figure 1 [2] demonstrates both the induction and explosion times as functions of temperature for various mixtures as measured in a static apparatus and shock tube. The induction period, as seen from Fig. 2 [2], can be defined quite easily from pressure or luminosity records as, for example, a time to an appreciable rise of the signal or to the inflection point, and $t_{\mathrm{e}}$ may be measured as a time to the maximum pressure or maximum luminosity. This definition of $t_{e}$ slightly underestimates it, especially when the signal rises slowly, since expansion of the gas may compensate for the signal growth due to the reaction. However for the majority of gasdynamic problems, especially for those relevant to shock wave-exothermic reaction coupling, it is the overall heat release time which is of importance. Figure 1 illustrates clearly that $t_{\text {ind }}$ is the principal constituent of the overall heat release time only at rather low temperatures and values of $t_{\text {ind }}$ above $10^{-4} \mathrm{~s}$, below the intersection point of the Arrhenius plots for $t_{\text {ind }}$ and $t_{\mathrm{e}}$ the heat release profile is no longer of an explosion type. A position of the intersection point depends on the pressure and temperature mainly due to a strong dependence of the induction period on these parameters, since $t_{\mathrm{e}}$ is affected by variations of pressure and temperature only slightly. The effective activation energy of $t_{\mathrm{e}}$ turned out to be only a few $\mathrm{kcal} / \mathrm{mol}$ and the pressure exponent of about $0.2-0.3$, whereas for the majority of hydrocarbons the effective activation energy of induction periods ranges between 30 and $50 \mathrm{kcal} / \mathrm{mol}$, while the pressure exponent changes between -0.7 and -1.0 . It should also be noted that unlike induction periods, $t_{\mathrm{e}}$ are affected (if so) by various chemical additives only insignificantly, which is accounted for by the fact that any additional production of reactive species in the system may change solely that part of the reaction which proceeds under conditions when the radical concentration is not too high. At later reaction stages the thermal production of radicals is so intense that only additives taken in amounts comparable with the concentrations of the initial reagents may influence the reaction rate.

The fact that the heat evolution rate during the explosion part of the temperature profile is slower than the rate which should be expected based on the kinetic parameters (activation energy and preexponential factor) derived from measured ignition delays is reflected in the term "recombination reaction stage" frequently used in detonation literature, though this definition is not precisely adequate (which follows from the considerations presented below). First, since the temperatures in a partially burnt gas are much higher than that at which the reaction has started, the Arrhenius exponents of all the elementary steps during the major fraction of $t_{e}$ are much less sensitive to temperature variations than at the beginning of the reaction (due to smaller $E / R T$ values). 

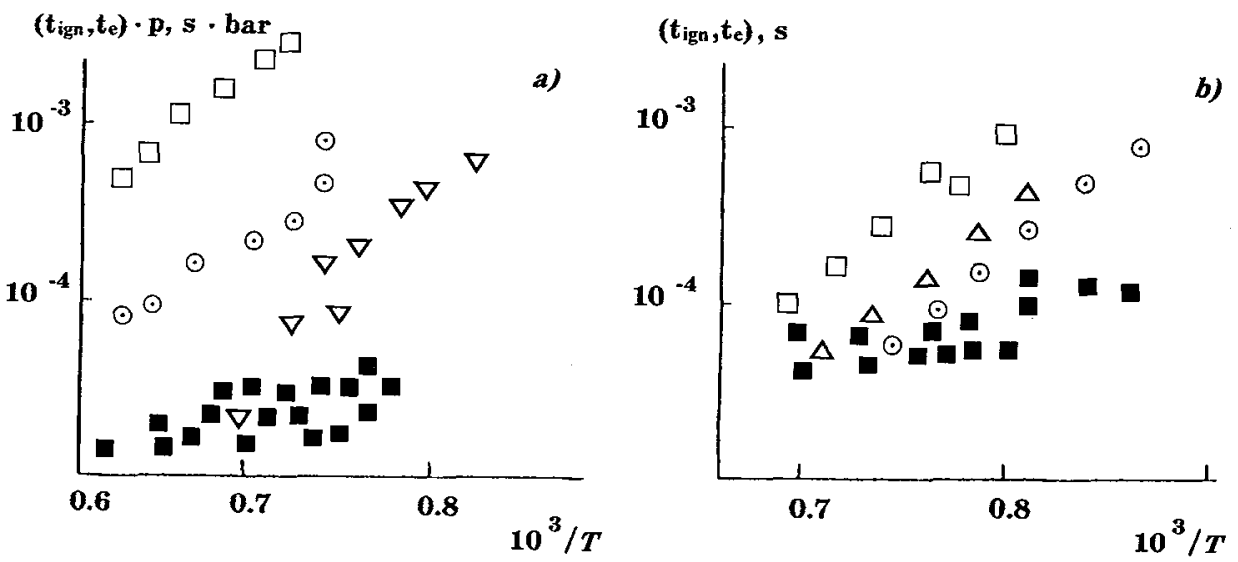

FIGURE 1. Arrhenius plots of the induction periods $t_{\text {ind }}$ and explosion times $t_{e}$ : (a) $\square 6 \%$ methane $+12 \% \mathrm{O}_{2}+82 \% \mathrm{Ar} ; 03.3 \%$ propane $+16.6 \% \mathrm{O}_{2}+82 \% \mathrm{Ar} ; \nabla 1.5 \%$ heptane $+16.5 \% \mathrm{O}_{2}+82 \% \mathrm{Ar} ; \mathbf{\square} t_{e}$ for all the mixtures; $(b) 4.3 \%$ propane $+21.3 \% \mathrm{O}_{2}+74.4 \%$ $\mathrm{N}_{2}: \square p=1$ bar; $\Delta p=2$ bar; $\circ p=5$ bar; $t_{e}$ for all the pressures.

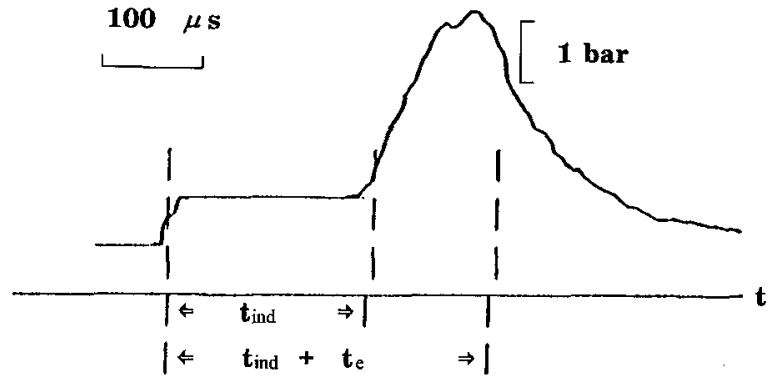

FIGURE 2. An example of pressure record behind the shock wave reflected from a rigid wall in a propane-air mixture. The initial flat portion corresponds to the induction period, and the rising portion is the heat release stage.

Second, the radical concentrations at these reaction stages are very high and therefore elementary reactions involving these reactive particles become dominant. This applies to recombination reactions as well, since it can easily be inferred from a mathematical analysis of the kinetic equations for chain, and even non-chain, reactions that the radical concentrations should pass through a maximum (which is the higher, the greater the contribution of the branching steps to overall radical production) in the course of the reaction, which means that there must exist a radical recombination stage. In the overwhelming majority of mixtures, this latter stage does not produce a significant contribution to the overall heat release, because the energy stored in the radicals formed constitute only a small fraction of the heat content of the reaction products.

That in an isothermal case the time histories of the radicals may peak at the early stage of the process is seen from very simple considerations. Indeed, even non-chain reactions start usually with decomposition of fuel (or oxidizer) molecules, that is with formation of radicals. The difference between chain and non-chain reactions consists in what happens further with the radicals formed: in non-chain reactions they recombine to produce species 
with a lower enthalpy of formation than the initial reagents, while in chain reactions they enter into chain propagation processes yielding both the end products and active intermediates (i.e. producing both heat and chemical energy). Of the principal importance is the point that the initial reagents produce radicals and atoms more easily than the end products since the latter have stronger bonds (otherwise the overall heat release will never be positive). Hence, the equilibrium radical concentrations for individual initial components of a combustible mixture may be higher than that for the end reaction products. The faster radical production in a chain branched reaction reduces the fraction of the active species recombined within the induction period, that is why the radical concentration overshoot in chain branched reactions is usually much (up to several orders of magnitude) higher than in other types of explosion reactions. However, under non-isothermal conditions, radical concentration overshoot can be observed only in branched chain reactions because of continuous decomposition of all the species present in the mixture with growing temperature.

Inasmuch as the activation energies of radical-radical and radical-intermediate reactions are lower than those of reactions involving the starting molecules, the overall effective activation energy will drop with the burnt fraction.

It follows from the aforesaid that the $E / R T$ factors at later reaction stages are lower than at its beginning and, hence, the role of the reactant depletion will be more prominent. The heat release profile becomes flatter with time due to all the aforementioned factors but not to radical recombination alone. Here we mention only chemical factors that flatten the heat release profile at the later reaction stages.

Now we consider how fast the recombination energy may be evolved. Let radicals be formed instantaneously in combustion products in amount of $R_{0}$. Then the characteristic concentration-decay time is $\left(R_{0} k_{\mathrm{r}} \mathrm{M}\right)^{-1}$, where $k_{r}$ is the recombination rate constant and $\mathrm{M}$ is the total concentration of species. This follows from the solution of the kinetic equation for quadratic radical recombination under the aforesaid initial condition. The value of $k_{\mathrm{r}}$ may be set equal to the frequency of triple collisions, i.e. to $10^{16} \mathrm{~cm}^{3} / \mathrm{mol} \cdot \mathrm{s}$. This yields for the recombination time a value of $10^{-12}\left(\mathrm{M} / \mathrm{R}_{0}\right) T^{2} / P^{2} \mathrm{~s}$. Here $P$ is pressure in atm. As mentioned above, concentrations $R_{0} / M$ lower than $1 \%$ may change the total thermal energy only less than by $1 \%$, therefore for practical estimates we may write that the recombination time should be less than $10^{-4} / P^{2}$. Thus, at pressures higher than $10 \mathrm{~atm}$ this time does not exceed $10^{-6} \mathrm{~s}$, i.e. recombination proceeds very fast in detonation systems. Although the above estimate is very rough, it applies to many practical situations. The main conclusion that can be drawn from this estimate is that variation of the radical concentrations throughout the explosion (intense) portion of the energy release profile should be more or less close to the quasi-steady one (because of very fast recombination). Hence, for radical concentration in the general case of chain branched reaction we may write at this reaction stage:

$$
\mathrm{R}=\left(k_{\mathrm{I}} \mathrm{A}_{2} / 2 k_{\mathrm{r}}\right)\left[1+\left(1+4 k_{\mathrm{I}} \mathrm{M} k_{\mathrm{r}} / k_{\mathrm{b}}{ }^{2} \mathrm{~A}_{2}\right)^{1 / 2}\right],
$$

where $k_{1}$ and $k_{b}$ are the rate constants of the initiation and branching reactions, respectively, and $\mathrm{A}$ is the fuel concentration. Thus the heat release rate at this stage is:

$$
\begin{gathered}
d q / d t=k_{\mathrm{pr}} q_{\mathrm{pr}} \mathrm{A}_{2} \mathrm{R}+k_{\mathrm{r}}^{2} \mathrm{R} q_{\mathrm{r}}+q_{\mathrm{r}} k_{\mathrm{l}} \mathrm{A}_{2}=\left(k_{\mathrm{pr}} q_{\mathrm{pr}}+k_{\mathrm{h}} q_{\mathrm{r}}\right) \mathrm{A}_{2} \mathrm{R}= \\
{\left[k_{\mathrm{b}} \mathrm{A}_{2} / 2 k_{\mathrm{r}}+\left(k_{\mathrm{I}} \mathrm{MA}_{2} / k_{\mathrm{r}}\right)\right]\left(k_{\mathrm{pr}} q_{\mathrm{pr}}+k_{\mathrm{b}} q_{\mathrm{r}}\right) \mathrm{A}_{2},}
\end{gathered}
$$

where $q_{i}$ are the reaction heats and subscript $p r$ signifies a chain propagation step. It is taken into account here that $q_{\mathrm{r}} \approx-q_{\mathrm{I}}$, so that term $k_{1} \mathrm{~A}_{2} \mathrm{M}\left(q_{\mathrm{r}}+q_{\mathrm{I}}\right)$ may be neglected not only because of smallness of the initiation rate constant but because of the small energy difference as well. This indicates that explosion proceeds at its later stages as a non-chain process with some effective rate constants and does not show any pronounced recombination stage. 
The absence of this stage is also shown by direct calculations of adiabatic explosion with detailed kinetics. Some of the results are presented in Figs. 3 and 4 , where the reacting systems are chosen deliberately to demonstrate the possible effect of the recombination stage on heat evolution. As expected from the above rough estimates, no profound recombination stage is observed, moreover, calculations contradict even the shock tube measurements, because the $t_{e}$ is always (even at high temperatures) much shorter than $t_{i n d}$. The only plausible explanation of this contradiction is that ignition behind shock waves (as well as behind detonation waves) is an essentially inhomogeneous process, that is, gasdynamic fluctuations that inevitably are generated behind shock waves due to flow interaction with the walls (or to detonation wave instability) lead to formation of a great many hot spots in the unburnt mixture. This hot spot ignition was repeatedly observed in shock tubes. Non-synchronous mixture ignition at various points changes the temperature-time history averaged over the tube cross section "stretching" its part corresponding to fast energy release (explosion proper) and decreasing is slope. This suggests that the major factor responsible for two-step kinetics of heat release behind detonation waves is hot-spot mixture ignition, which makes it different from calculations based only on detailed chemical mechanisms under homogeneous conditions. Although hot spot ignition does not affect appreciably the kinetic parameters derived from the ignition delays measured in shock tube experiments because mixtures used in these experiments are highly diluted. But in detonable mixtures this may not be the case, therefore direct transfer of the experimental data obtained in shock tubes to detonation waves requires special consideration. The high scatter of the shock tube data on ignition delays is the well recognized fact. It should naturally be ascribed to inhomogeneous ignition of the mixture. And yet, one can not expect strong deviations of the ignition delays measured in reactive mixtures from those corresponding to a homogeneous reaction, because a direct comparison of the ignition delays measured in individual stream tubes behind the lead shock wave of a spinning detonation with shock tube measurements and chemical kinetic calculations demonstrates their consistency.

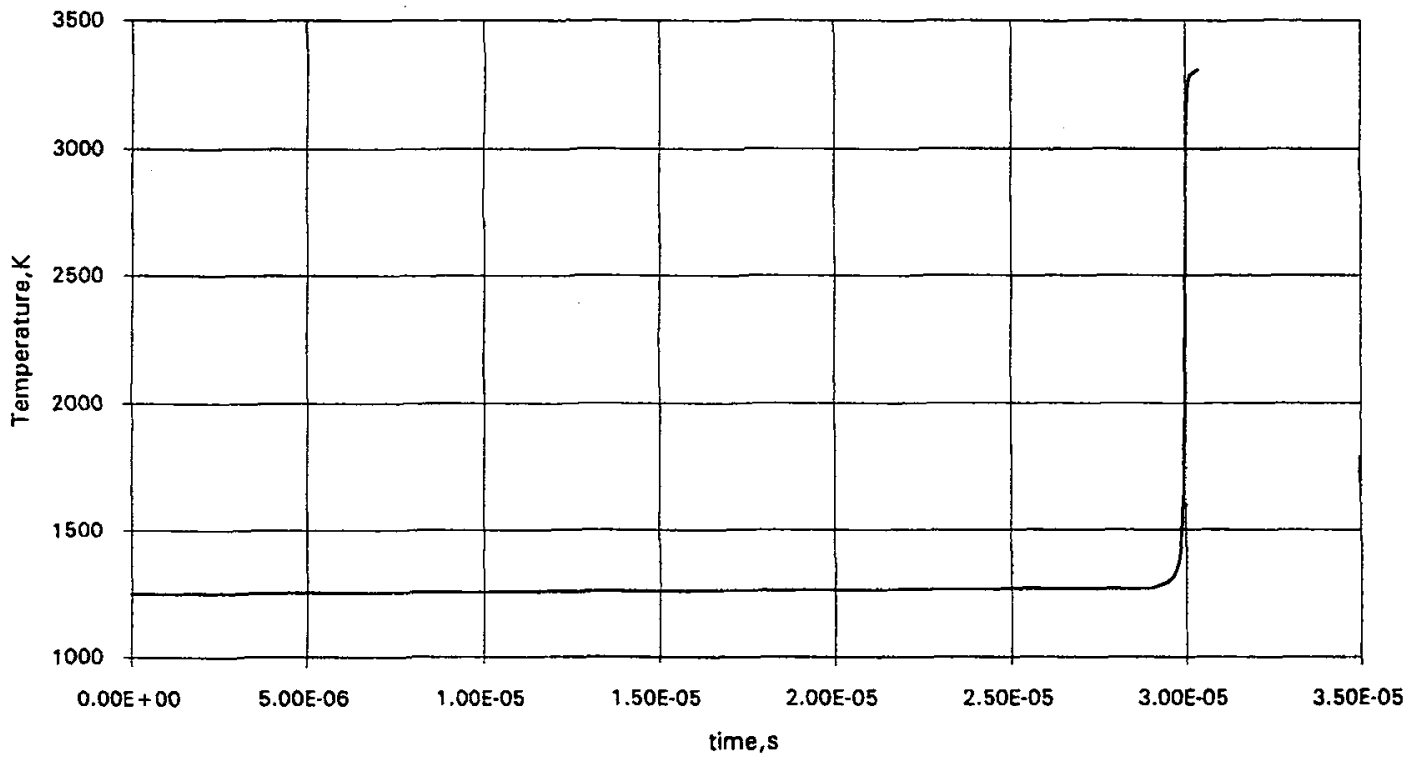

FIGURE 3. Temperature - time history for explosion of an $2.15 \mathrm{H}_{2}+\left(\mathrm{O}_{2}+3.76 \mathrm{~N}_{2}\right)$ mixture at $T_{0}=1250 \mathrm{~K}$ and $p_{0}=28.3 \mathrm{bar}$. 


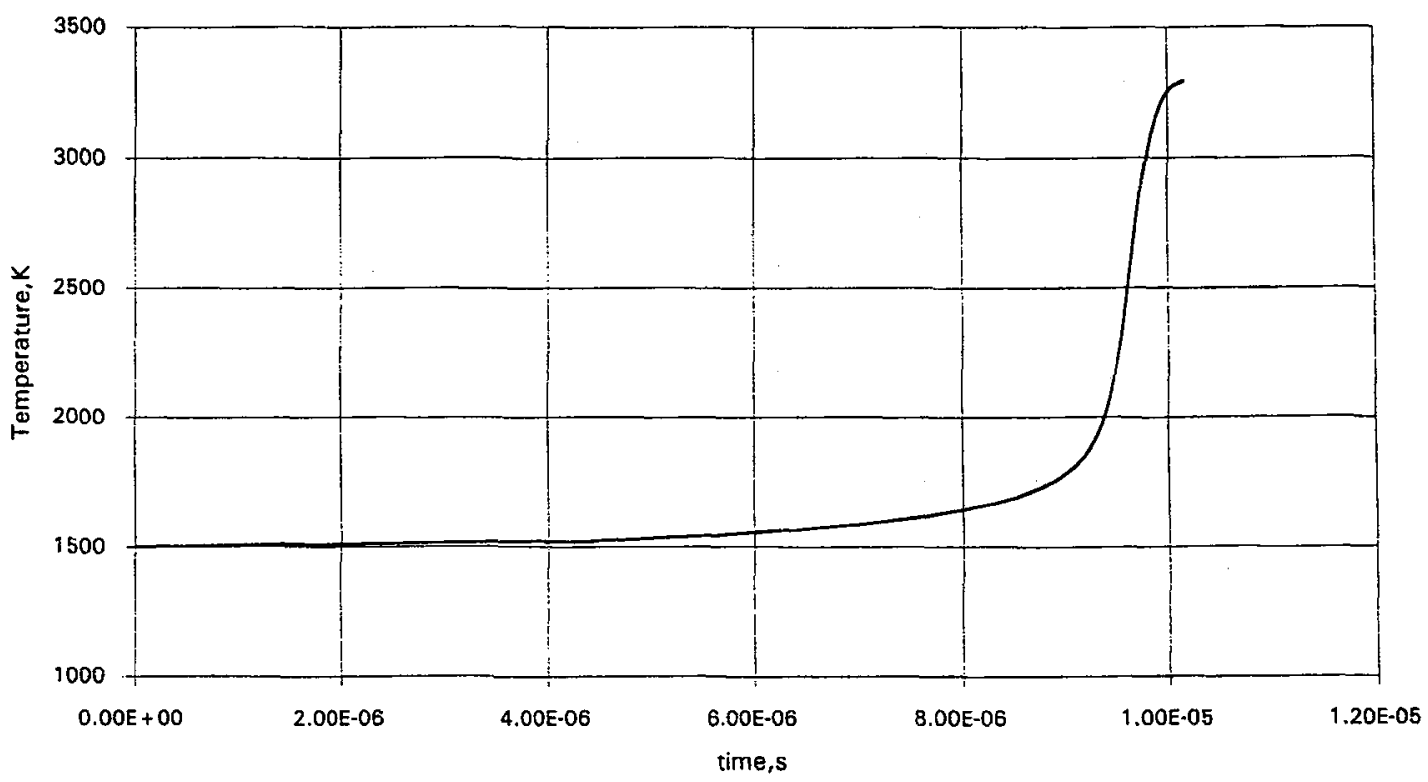

FIGURE 4. Temperature - time history for explosion of an $\mathrm{C}_{3} \mathrm{H}_{8}+5\left(\mathrm{O}_{2}+3.76 \mathrm{~N}_{2}\right)$ mixture at $T_{0}=1500 \mathrm{~K}$ and $p_{0}=28.3 \mathrm{bar}$.

Thus, all the onedimensional detonation calculations, in which the heat release rate is averaged over mixture volume, should be performed with an at least realistic reaction kinetics, e.g. that measured in shock tube experiments where the whole energy release profile is monitored. Hence, calculations with a detailed chemical kinetics are meaningful only when no averaging procedure is used, i.e. within three- or two-dimensional hydrocodes in which local temperature fluctuations are automatically taken into account.

However, detailed reaction schemes are inconvenient for complicated threedimensional gasdynamic calculations because they bring about computational difficulties (which turns out unsurmountable in many cases), on the other hand, they are inapplicable to one-dimensional calculations. The most rational way to cope with this problem is to derive global kinetic equations, deriving them either from detailed reaction schemes for threedimensional calculations or from shock tube measurements for onedimensional calculations. Below we illustrate both approaches.

The basic parameter for comparison with experiment is the ignition delay which is nearly identical in both cases. The main idea of further calculations is simulation of the entire heat release profile by expressions valid for the delay of thermal explosion. As follows from the theory of thermal explosion, ignition delay is related to the heat release rate at the zero-time conditions:

$$
t_{\text {ind }}=c_{p} R T_{0}^{2} /(E Q w)
$$

where $c_{p}$ is the specific heat, $E, Q$, and $w$ are the effective activation energy, heat, and relative rate of the reaction, which are not necessarily the same throughout the temperature interval considered. This is illustrated in Fig. 5, where ignition delays for various hydrogenair mixtures are summarized. Hence, to find the real reaction rate, one needs to express $w$ via $t_{\text {ind }}$, introduce the dependence of $w$ on concentrations of the reagents, and assume the temperature in the expression for $t_{\text {ind }}$ variable. This procedure hopefully will allow one to 


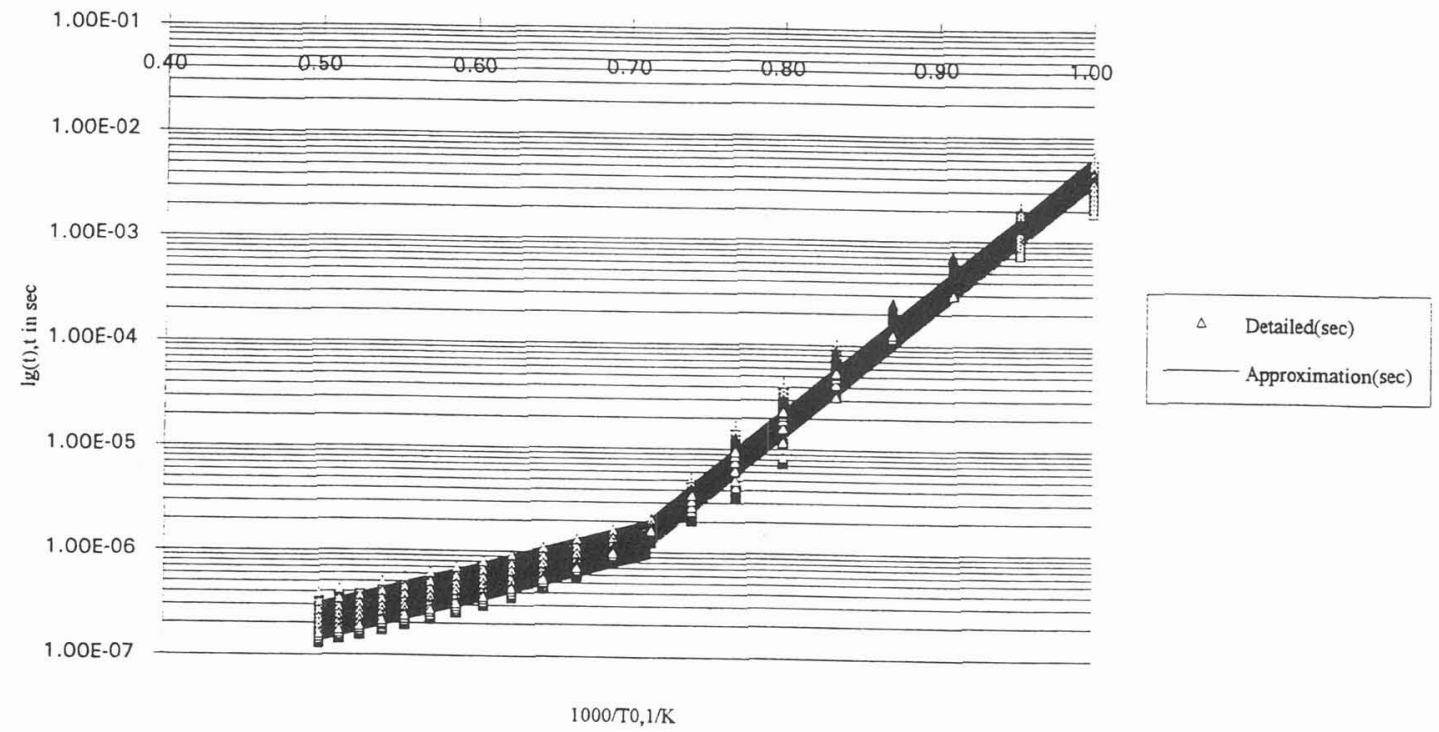

FIGURE 5. Calculated ignition delays of various hydrogen-air mixtures. Symbols are calculations by the detailed reaction mechanism, lines are calculations by the approximating equations. The concentartion and pressure ranges are: $19-59 \% \mathrm{H}_{2}$ and $15-35$ bar, respectively.

use measured induction periods, at least in the region of temperatures and pressures where they could unambiguously be defined, to more or less adequately describe the reaction course. As far as the detailed kinetic calculations are concerned, Fig. 6 compares the exact solution and the temperature profile calculated by the approximate formula. Although the shapes of the curves differ significantly, the most important characteristic - the overall reaction time fits very well. It should be emphasized that even with the effective reaction orders much less than those derived from shock tube experiments the global formula yields lower slopes of the explosion part of the temperature profile, which indicates that any two stage reaction mechanisms are not of the purely chemical nature.

In order to take into account flattening of the effective heat release profile during later stages of an adiabatic thermal explosion (in onedimensional calculations) one may use a parameter which is tacitly assumed not to affect the course of the reaction within the induction period in a mixture with a high chemical energy stored. This parameter is the effective reaction order. As follows from the above considerations, the structure of the final expressions describing both the induction and intense-heat-release reaction stages is not very different for thermal and chain-thermal explosions (except for chain branched reactions with a very low contribution of recombination reactions to the radical concentration variation during the induction period). Thus there is some hope to write a single heat release equation for both stages but with some effective reaction order which will not change the expression for the induction period but will enable one to fit the "explosion part" of the heat release profile to measured $t_{\mathrm{e}}$. In accord with the above hypothesis one may express the reaction rate as follows:

$$
-d \mathrm{~A}_{2} / d t=c_{\mathrm{pRT}}{ }^{2}\left(\mathrm{~A}_{2} / \mathrm{A}_{2}{ }^{0}\right)^{\mathrm{n}} /\left(Q E t_{\text {ind }}\right) .
$$




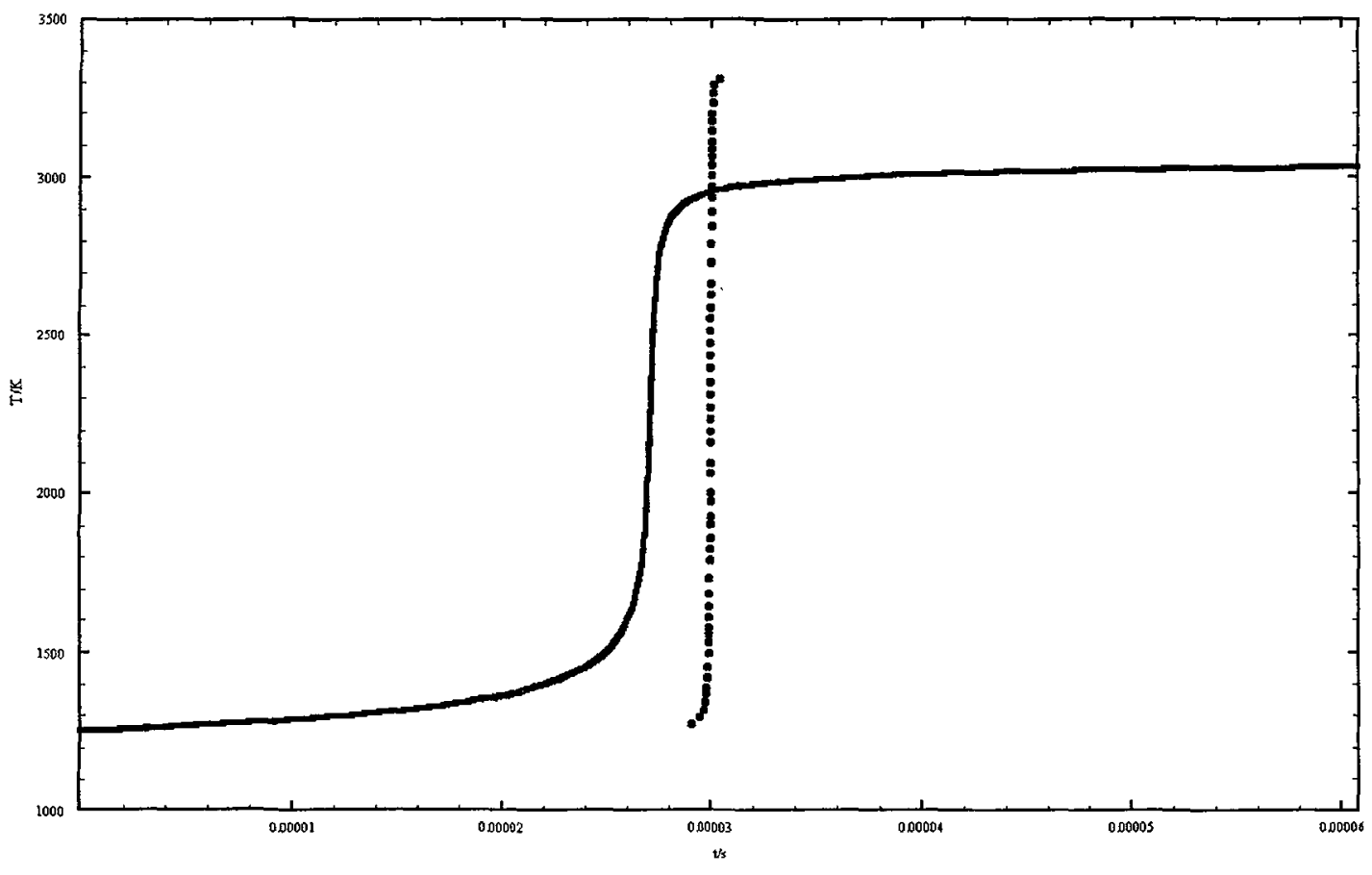

FIGURE 6. Comparison of the temperature profiles calculated by the detailed reaction mechanism and approximate kinetic equation. The conditions are same as in Fig. 3.

Here the temperature in the experimental expression for the ignition delay is assumed to be equal to its instantaneous value (the reagent concentrations in the empirical equation for $t_{i g n}$ being retained equal to the initial ones), and factor $\left(A_{2} / A_{2}{ }^{0}\right)^{n}$ is introduced to allow for the change in the reaction rate at later reaction stages.

The effective reaction order, which allows one to fit the calculated and measured $t_{i g n}$ and $t_{e}$ with a fairly good accuracy turned out to be very weakly dependent on the type of a hydrocarbon fuel, thus for propane- and ethylene- air mixtures it is three, and for methane-air mixtures it is 4.5 .

As for chain branched reactions with low efficiency of the chain termination steps, it should be emphasized that this latter approach is hardly to be applicable to description of the whole heat release profile, at least with some realistic activation energies and rate constants. For this reason the two-term formula for the reaction rate [2] could be recommended in this case.

\section{CRITICAL DETONATION PARAMETERS AND THE WAVE STRUCTURE}

In order to check validity of the ZND model with effective reaction kinetics we attempted to predict a critical diameter for detonation propagation in tubes and minimum energy of detonation initiation in tubes for a propane-air mixture. Onedimensional calculations were essentially based on the empirical equation for ignition delays and effective reaction order found also from shock tube measurements. Heat and momentum transfer to the tube walls were taken into account.

Figures 7a and 7b shows "go" and "no go" calculations of detonation initiation by two plane point explosion energies evolved instantaneously in the tube. The calculated minimum initiation energy of $4.0 \mathrm{MJ} / \mathrm{m} 2$ does not differ very much from its experimental counterpart 


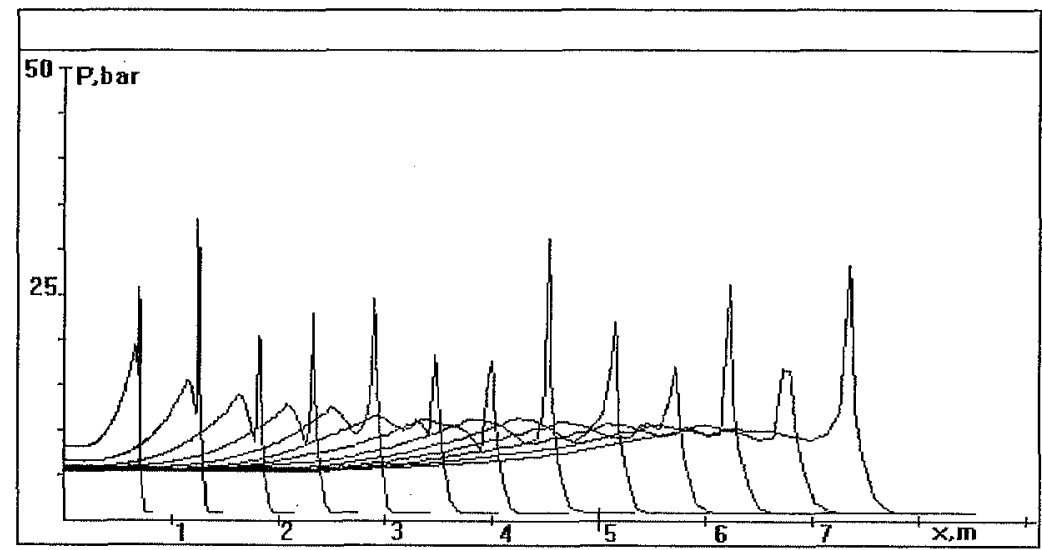

a.

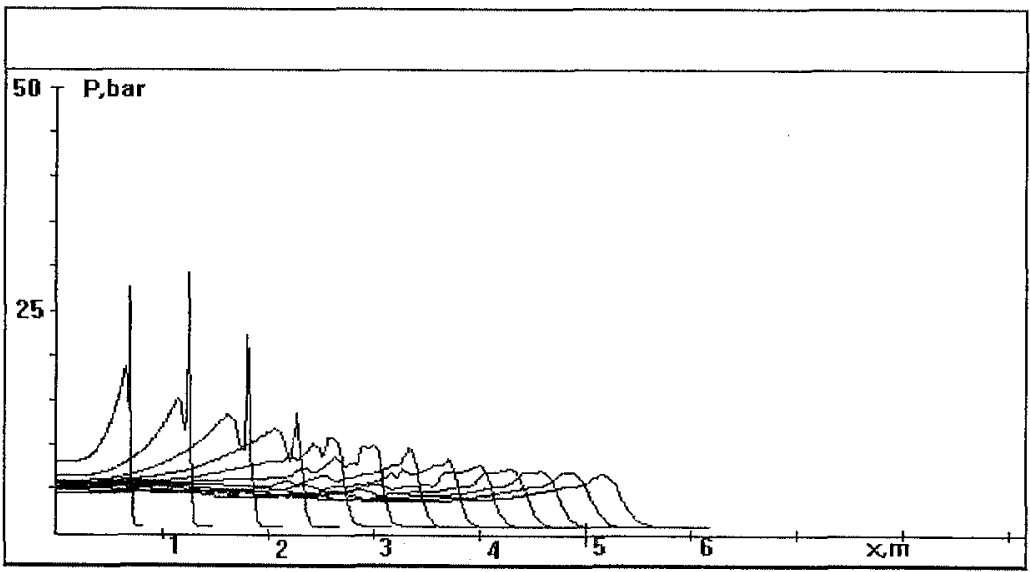

b.

FIGURE 7. Initiation of detonation in a propane-air mixture (point plane explosion): (a) go, $E_{\text {init }}=4.1 \mathrm{MJ} / \mathrm{m}^{2}(b)$ no go, $E_{\text {init }}=3.9 \mathrm{MJ} / \mathrm{m}^{2}$.

equal to $2.7 \mathrm{MJ} / \mathrm{m} 2$ under similar conditions. The estimated critical tube diameter $(17 \mathrm{~mm})$ is also consistent with measurements $(19 \mathrm{~mm})$.

Calculations revealed also importance of the longitudinal detonation wave fluctuations on the dependence of the concentration limits of detonation on tube diameter. Greater fluctuations of the reaction zone length at larger tube diameters do not allow it to grow unboundedly with the diameter, that is at large tube diameters the lean detonation limit changes only insignificantly, which is in full agreement with experimental observations.

Thus, we can conclude that ZND model can adequately predict critical parameters of detonation waves when the effective reaction kinetics is properly chosen (i.e. takes into account hot spot ignition). It is not surprising, because the critical parameters are governed by the longest reaction zone parts in which the induction period dominates and the hot spot effect, changing mainly the zone of intense energy evolution, is not very critical.

The lack of experimental data on the ignition delays makes often researchers use detonation cell sizes $\lambda$ as substituents. Although there is some correlation between the 
reaction zone and $\lambda$, one should be very cautions when deriving kinetic parameters from measured $\lambda$ values. As we demonstrate below, the major reaction is completed at distances less than the longitudinal detonation cell size $(b)$ and there is no grounds to believe that the average reaction zone constitutes a definite fraction of $b$.

Unlike the appropriate graph of ignition delays, the Arrhenius plot of $b$ is highly nonlinear. This is illustrated in Fig. 8, where $\log b$ measured in propane-oxygen mixtures

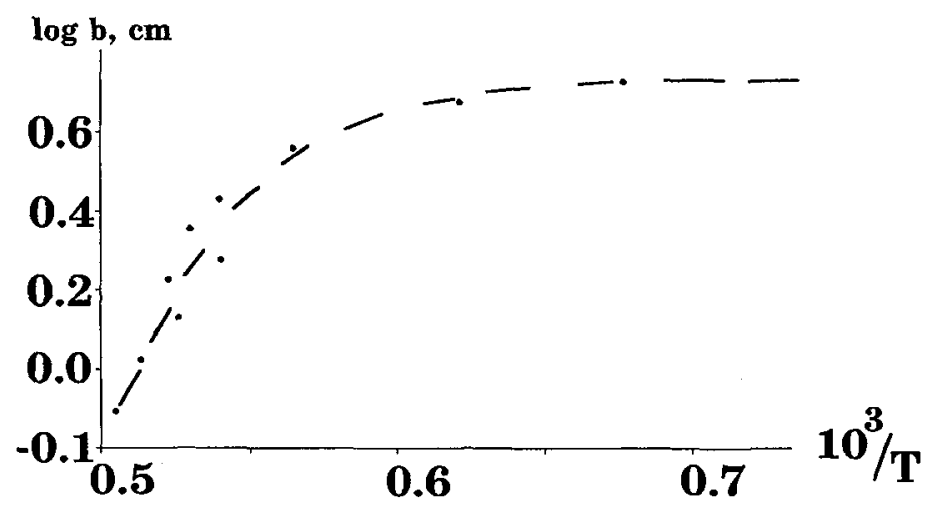

FIGURE 8. Arrhenius plot of the longitudinal detonation cell size for $\mathrm{C}_{3} \mathrm{H}_{8}-\mathrm{O}_{2}-\mathrm{N}_{2}$ mixtures (with variable $x$ ).

containing different amounts of nitrogen is plotted as a function of the average temperature behind the plane lead shock wave propagating at a detonation velocity. This means that $b$ can not be recommended as a precise kinetic parameter.

In contrast to critical detonation characteristics, the ZND model with the abovediscussed heat release kinetics is inapplicable to describing the behavior of the averaged parameters behind a detonation wave. Density profiles exhibit the highest deviation from the traditional ZND model. Indeed, Fig. 9 shows measurements of various parameters behind a detonation wave in a propane/oxygen/nitrogen/krypton mixture at atmospheric pressure in a tube $50 \mathrm{~mm}$ i.d.. The detonation velocity is $1569 \mathrm{~m} / \mathrm{s}$ and the transverse detonation cell size is $17-18 \mathrm{~mm}$. The X-ray absorption signal is the only truly averaged measured parameter. Instead of the gradually decaying signal profile (predicted by the ZND model), we see a curve with two minima. The first absorption peak should be assigned to the von Neumann spike within which the major reaction is completed. This is supported by the $\mathrm{OH}$ emission and ionization current signals that peak also within this period of time (that is, intense generation of excited $\mathrm{OH}$ radicals and chemi-ions ceases). The second peak is also seen on all the records. Most likely, this peak corresponds to collision of flappy transverse waves (that is to the end of detonation cells), which is confirmed by measurements of their size. The rise of all the signals after the second minimum is not quite clear as yet. There could be several factors leading to this rise, which we discuss below.

Going back to the first portion of the records, we may state that, within the ZND model, this flow portion has an averaged sonic plane slightly upstream of the absorption signal minimum. The gas density grows after this minimum because part of the kinetic energy of transverse motion is converted into the internal energy, which is equivalent to an additional heat release in the flow averaged over the tube cross section. This effective heat release downstream of the averaged sonic plane (where the flow is supersonic with respect to the lead shock front) produces a compression wave rising the flow parameters (density, temperature, local dissociation and ionization degrees, and pressure), which is clearly shown by all the experimental records. This compression wave is followed by a rarefaction wave (after the second sonic plane). 

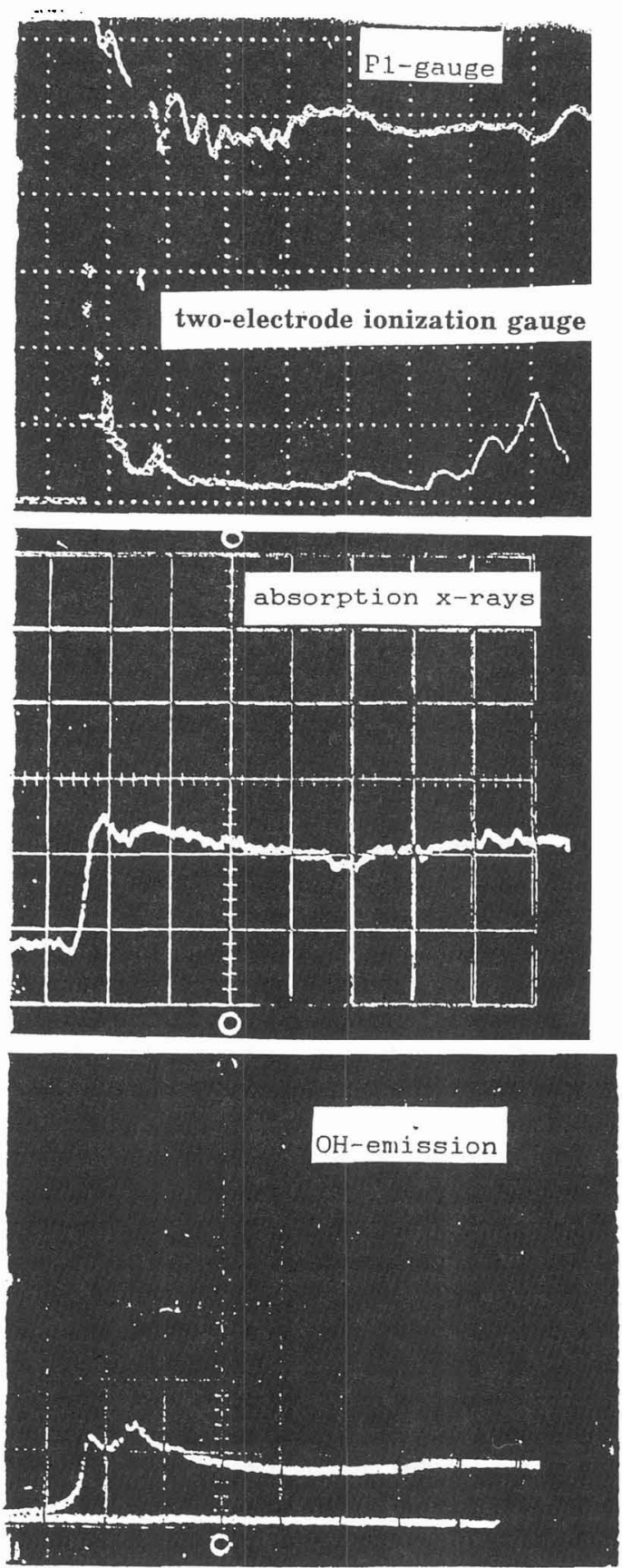

FIGURE 9. Records of pressure, ionization current, X-ray absorption, and $\mathrm{OH}$ emission behind a detonation wave in a propane - oxygen - nitrogen -krypton mixture. 
The final rise of the effective temperature and density after the second minimum (see the ionization current and $\mathrm{OH}$ emission) could be attributed to: (a) burning out of tiny freshmixture blobs formed in three-dimensional detonation waves (and revealed in gasdynamic calculations); (b) continuing redistribution of the energy between the transverse gasdynamic motion and internal degrees of freedom and between hotter stream tubes and colder ones; and (c) boundary layer formation. Which of them is dominant can not be decided without additional investigations.

\section{SUMMARY}

The above analysis of experimental observations of detonations in gases suggests that the ZND model applied in its traditional one-dimensional form can be used to predict some critical detonation waves (critical diameters, minimum energy of detonation initiation), provided the appropriate kinetics of heat release behind the lead shock wave allowing for hot spot ignition is incorporated in the calculation model.

Calculations of the quasi-onedimensional detonation wave structure requires a more sophisticated heat release model, because redistribution of the energy between the transverse gas motion and internal degrees of freedom and longitudinal flow changes dramatically this structure resulting in a flow with at least two sonic planes (instead of one in the ZND model) behind the lead shock wave.

\section{ACKNOWLEDGEMENT}

The research described in this publication was made possible in part by Grant No. M3G000 from the International Science Foundation.

\section{REFERENCES}

1. V.A. Levin, V.V. Markov, and S.F. Osinkin, Khimicheskaya Fizika, v. 3, No. 4, pp. 611-614, (1984)).

2. A.A. Borisov et al., Progress in Astronautics and Aeronautics, v.114, pp. 124-139, (1988). 